\title{
GESTÃO DA ENFERMAGEM EM HOSPITAL GERAL PÚBLICO ACREDITADO NO ENFRENTAMENTO DA PANDEMIA POR COVID-19
}

Paula Maria Corrêa de Gouveia Araujo $0^{1,2}$ Elena Bohomol ${ }^{2}$

Tereza Aparecida Benjamim Teixeira ${ }^{1}$ https://orcid.org/0000-0002-8930-2931 https://orcid.org/0000-0002-7196-0266

http://orcid.org/0000-0002-7283-0893

Objetivo: Relatar a experiência vivenciada pela gestão de enfermagem de um hospital geral público acreditado e apresentar as principais mudanças na estrutura institucional para o enfrentamento da pandemia. Método: Relato de experiência em uma Organização Social de Saúde de parceria com governo estadual. Trata-se de um hospital geral de nível terciário com 247 leitos. Resultados: As principais mudanças estruturais e de processos de trabalho ocorridas durante o periodo de 13 de março a 23 de abril de 2020 foram: Implantação de um Comitê de Crise; programação de treinamentos institucionais; medidas administrativas voltadas ao cancelamento de cirurgias eletivas; disponibilização de equipamentos de proteção individual; mudanças no fluxo de entrada do paciente com suspeita da COVID-19; mudanças nas características das unidades; incremento no número de leitos; contratação de pessoal; e apoio para equipe. Conclusão: Destaca-se a importância da equipe de enfermagem e seu preparo para gerenciar novos espaços, alocar recursos e treinar a equipe no atendimento direto ao paciente.

Descritores: pandemia; COVID-19; enfermagem; infecções por coronavirus.

\section{NURSING MANAGEMENT IN AN ACCREDITED PUBLIC GENERAL HOSPITAL IN THE RESPONSE TO THE COVID-19 PANDEMIC}

Objective: Reporting the experiences of the nursing management team of an accredited Brazilian public general hospital and the main institutional changes made to face the COVID-19 pandemic. Method: An experience report about a Social Healthcare Organization that has a partnership with the São Paulo state government. This is a 247-bed tertiary care hospital. Results: During the period between March 13 and April 23 2020, the main changes made to the institution and its work practices were: Implementing a Crisis Committee; scheduling institutional trainings; managerial measures concerning the cancellation of elective surgeries; providing personal protective equipment; changes in the admission of patients with suspected COVID-19; changes in the characteristics of the hospital unit; an increase in the number of beds; hiring new personnel; and supporting the current staff. Conclusion: This highlights the importance of the nursing team and how prepared it is to manage new rooms, allocate resources, and get the staff ready for the direct care of patients.

Descriptors: pandemic, COVID-19; nursing; coronavirus infections.

\section{GESTIÓN DE ENFERMERÍA EN UN HOSPITAL GENERAL PÚBLICO ACREDITADO EN EL ENFRENTAMIENTO DE LA PANDEMIA DA COVID-19}

Objetivo: Reportar la experiencia de la gestión de enfermería de un hospital general público acreditado brasileño y presentar los principales cambios en la estructura institucional para enfrentar la pandemia da COVID-19. Método: Registro de experiencia en una Organización Social de Salud en colaboración con el gobierno del Estado de São Paulo. Se trata de un hospital general de nivel tercer nivel con 247 camas. Resultados: Los principales cambios estructurales y en los procesos de trabajo ocurridos en el periodo entre el 13 de marzo y el 23 de abril fueron: La implantación de un Comité de Crisis; programación de prácticas institucionales; medidas administrativas dirigidas a la cancelación de cirugias electivas; disponibilidad de equipos de protección individual; cambios en el flujo de ingreso de pacientes con sospecha da COVID-19; cambios en las características de la unidad hospitalaria; incremento en el número de camas; contratación de personal; y apoyo al equipo. Conclusión: Se destaca la importancia del equipo de enfermería y su preparación para gestionar nuevos espacios, alocar recursos e instruir el equipo para la atención directa al paciente.

Descriptores: pandemia; COVID-19; enfermaría; infecciones por coronavirus.

IHospital Santa Marcelina de Itaquaquecetuba, São Paulo, SP.

${ }^{2}$ Universidade Federal de São Paulo - UNIFES, São Paulo, SP.

Autor Correspondente: Paula Maria Corrêa de Gouveia Araujo E-mail: paulamcgouveia@gmail.com

Recebido: 30/4/2020

Aceito: $28 / 5 / 2020$ 


\section{INTRODUÇÃO}

$\mathrm{Na}$ China, em dezembro de 2019, a província de Hubei, tornou-se o centro de um surto de pneumonia de causa desconhecida, que despertou intensa atenção não apenas na China, mas internacionalmente. As autoridades de saúde chinesas fizeram uma investigação imediata para caracterizar e controlar a doença, incluindo isolamento de pessoas suspeitas de ter a doença, monitoramento próximo dos contatos, coleta de dados epidemiológicos e clínicos dos pacientes e desenvolvimento de procedimentos de diagnóstico e tratamento. Em sete de janeiro de 2020, os cientistas chineses haviam isolado um novo coronavírus (CoV) de pacientes em Wuhan ${ }_{(1)}$

O coronavírus da sindrome respiratória aguda grave (SARS-CoV) e o coronavírus da síndrome respiratória do Oriente Médio (MERS-CoV) são dois vírus altamente transmissiveis e patogênicos que surgiram nos seres humanos no início do século XXI. É provável que ambos os vírus tenham se originado em morcegos, e os coronavírus geneticamente diversos relacionados ao SARS-CoV e MERSCoV foram descobertos em morcegos em todo o mundo(2). Até então, neste momento, ainda estava uma situação de epidemia. Esse novo coronavírus é responsável por causar a doença COVID-19(3)

Em 16 de janeiro, foi notificada a primeira importação em território japonês. No dia 21 de janeiro, os Estados Unidos da América citaram seu primeiro caso importado. Em 30 de janeiro, a Organização Mundial de Saúde (OMS) declarou a epidemia uma emergência internacional (4)

No Brasil, foi instituída a Portaria no 356 do Ministério da Saúde (MS), que dispõe sobre a regulamentação e operacionalização do disposto na Lei no 13.979 , de seis de fevereiro de 2020, que estabelece as medidas para enfrentamento da emergência de saúde pública de importância internacional decorrente da COVID-19 (5). Apesar de ter sido instituído a portaria do MS em fevereiro, foi somente em março que se intensificou o assunto da doença na mídia do Brasil. Em 11 de março de 2020, à COVID-19 foi caracterizada pela OMS como uma pandemia(3)

Aatuaçãonocontroleeprevençãodedoençasinfecciosas, doenças transmissiveis e nos danos que podem ser causados durante o atendimento aos pacientes, são funções dos profissionais da saúde e também da equipe de enfermagem. No entanto, os profissionais de saúde envolvidos no cuidado de pessoas infectadas com COVID-19, sejam daqueles que apresentam uma evolução lenta da doença seja dos que têm suas condições de saúde agravadas por causas subjacentes, podem apresentar distúrbios emocionais que colocam sua saúde, bem estar psicológico e saúde mental em risco, afetando seu desempenho profissional ${ }^{(6)}$

Por outro lado, além do agravamento clínico em muitos pacientes, os profissionais que os atendem estão continuamente sujeitos a uma situação extrema e estressante, devido a múltiplos fatores como alarme social, falta de recursos, saturação dos serviços, incerteza, etc., transformando-o em segunda vítima deste fenômeno (7). As conclusões de um estudo apontam que a situação de trabalho necessita ser vista à luz de políticas públicas para que o trabalho em Enfermagem seja feito em condições dignas e satisfatórias ${ }^{(8)}$.

Em virtude deste momento de pandemia, as instituições hospitalares têm transformado sua estrutura e seu processo de trabalho para possibilitar os melhores resultados para o atendimento ao paciente e para a manutenção física e psicológica da equipe da linha de frente. Ainda que haja um dinamismo na tomada de decisões, as ações têm a intenção para que as instituições cumpram o seu papel para assistir aos que necessitam sem negligenciar as demandas que surgem a cada momento ${ }^{(9)}$.

Assim, este artigo tem o objetivo de relatar a experiência vivenciada pela gestão de enfermagem de um hospital geral público acreditado e apresentar as principais mudanças na estrutura institucional para o enfrentamento da pandemia.

\section{MÉTODO}

Relato de experiência sobre a vivência da gestão de enfermagem no momento de pandemia por COVID-19, em uma Organização Social de Saúde (OSS), de parceria com governo estadual. Trata-se de um hospital geral de nível terciário, sendo também centro de referência para o trauma e maternidade de alto risco, localizado na região de Itaquaquecetuba da Grande São Paulo eatende os municípios de Itaquaquecetuba, Arujá, Ferraz de Vasconcelos, Santa Isabel, Guararema, Mogi das Cruzes, Poá, Suzano, Biritiba Mirim e Salesópolis. Esta Unidade foi inaugurada em março de 2000, possui 247 leitos distribuídos em 10 unidades de atendimento, com 10 leitos de Unidade de Terapia Intensiva (UTI), 6 salas de cirurgia e pronto socorro.

A instituição tem a certificação de qualidade da Organização Nacional de Acreditação (ONA) desde 2009 e trabalha regularmente para a manutenção e melhoria dos seus processos de trabalho.

O Serviço de Enfermagem é composto por 592 profissionais de enfermagem (17,73\% enfermeiros, $23,65 \%$ técnicos, 58,61\% auxiliares de enfermagem) com jornada de 36 horas semanais. Este dimensionamento corresponde ao número de pessoal anterior ao aumento de quadro que ocorreu no dia um de abril de 2020. A estrutura organizacional do serviço é representada por uma gerente de enfermagem, oito supervisores de enfermagem, uma enfermeira supervisora na educação continuada, 582 profissionais que atuam nas unidades de atendimento ao 
paciente. O período de relato corresponde de 13 de março a 23 de abril de 2020 .

\section{DESCRIÇÃO DA EXPERIÊNCIA}

Frente a Pandemia decretada pela Organização Mundial de Saúde (OMS), para organização do atendimento aos pacientes que necessitam de assistência em casos suspeitos e confirmados para COVID-19, a instituição tomou algumas medidas a partir de 13 de março de 2020, relatadas a seguir:

1. Implantação de um Comitê de Crise com 10 pessoas, representadas inicialmente pelos seguintes membros: diretor administrativo, diretora técnica, gerente administrativo, gerente de apoio, gerente de enfermagem, enfermeira do núcleo interno de regulação (NIR), supervisora de enfermagem da educação continuada, coordenador da qualidade, supervisor de enfermagem do Pronto Socorro, supervisores de enfermagem do plantão noturno e médico infectologista. A finalidade desse comitê era $\circ$ de estudar estratégias para $\circ$ atendimento, como: local de atendimento do paciente com suspeita, definição de fluxos, definição de escala de contingência dos profissionais, treinamento dos profissionais, dentre outras demandas surgidas. As reuniões ocorriam diariamente na sala de reuniões e para facilitar a comunicação deste comitê foi criado um grupo em um aplicativo multiplataforma de mensagens instantâneas (whatsApp). Os encontros tinham duração de aproximadamente 30 minutos e as estratégias a serem implementadas eram direcionadas aos membros que tinham a responsabilidade de implantá-las de imediato sendo responsáveis pelas mesmas a partir de então. Um exemplo, dos dados que seriam atualizados diariamente é o Monitoramento de internações de paciente com suspeita COVID-19.

2. Programação de treinamentos institucionais: $O$ primeiro treinamento foi entre 18 a 24 de Março para os profissionais de todas as áreas de atuação do hospital, ministrado pelas enfermeiras do serviço de controle de infecção hospitalar (SCIH), com o seguinte conteúdo sobre COVID - 19: o que é a doença, sinais, uso de equipamentos de proteção individual (EPI) e fluxo de atendimento. O treinamento tinha duração de 30 minutos e foi ministrado para os profissionais no auditório do hospital nos períodos da manhã, tarde e noite e atingiu aproximadamente 300 pessoas.

O segundo treinamento ocorreu entre 16 a 23 de abril para os profissionais de enfermagem e fisioterapia que estão lidando com os pacientes com suspeita ou confirmados para COVID-19. Foi agendado pelo serviço de educação continuada e ministrado pelas enfermeiras do SCIH. O tema foi: Paramentação com burcas. O treinamento atingiu 118 profissionais e foi realizado nas Unidades assistenciais, utilizando a metodologia de aula demonstrativa.

3. Medidas administrativas voltadas ao cancelamento de cirurgias eletivas: Essas medidas foram realizadas, devido a necessidade de disponibilizar o ambulatório cirúrgico para a entrada dos pacientes com suspeita da COVID-19. 4. Equipamentos de proteção individual (EPI): Foi intensificada a disponibilização e a supervisão da utilização de máscaras simples, máscaras N95, capote, aventais, óculos e higiene das mãos. Também foi entregue para todos os profissionais que atuam no hospital uma almotolia de álcool gel e orientado a possibilidade de reabastecimento sempre que necessário.

5. Mudanças no fluxo de entrada do paciente com suspeita da COVID-19: O atendimento normal deste tipo paciente era feito no Pronto Socorro e após a decisão do Comitê de Crise foi direcionado para o ambulatório. Tal medida visa facilitar o fluxo dos demais pacientes que passam pela classificação de risco e contribui para priorizar $\circ$ atendimento deles. $\bigcirc$ ambulatório foi montado com os recursos materiais e humanos já existentes na Instituição. $O$ atendimento era de 24 horas. Todos os pacientes que tinham indicação de internação, era comunicado ao núcleo interno de regulação para o direcionamento do leito.

6. Mudanças nas características das unidades: Como administração do cenário, houve a desocupação gradativa dos leitos de internação na Clínica Médica, posteriormente na Unidade Cirúrgica de Ortopedia e Ginecologia, para receberem os pacientes com suspeita, disponibilizando aproximadamente 26 leitos para esse atendimento. Alguns quartos são de quatro, dois e um leito (isolamento). Apesar da Unidade de Clinica Médica manter uma ocupação de 25 a 50\% neste período, foi mantido o mesmo dimensionamento de pessoal. Houve restrição na liberação de visitas e acompanhantes.

7. Incremento no número de leitos: O hospital inaugurou no início de Abril, uma Unidade de Cuidados Intensivos (UTI II) com 12 leitos para os atendimentos de pacientes graves com COVID-19. A Unidade é uma iniciativa do Governo do Estado de São Paulo e do MS. Os recursos disponibilizados para o atendimento de cada paciente contavam com: cama, respirador e bomba de infusão. Uma equipe composta por médicos, fisioterapeutas, enfermeiros e técnicos de enfermagem, para este atendimento. Os leitos foram ocupados gradativamente. 8. Contratação de pessoal: A diretoria administrativa do hospital autorizou a contratação de 29 vagas de técnicos de enfermagem e seis enfermeiros para garantir o atendimento de enfermagem da Unidade nas 24 horas. 
E, pensando em valorizar os talentos da Instituição, foi aberto um processo seletivo de promoção interna emergencial para preenchimento das vagas de técnicos de enfermagem, porém ocorreram negativas em relação à oferta da vaga para atuação na UTI II, provavelmente devido ao receio de atuar com pacientes suspeitos ou confirmados para COVID-19

9. Apoio para a equipe: As ações acima, como disponibilização de EPIs, treinamentos, dimensionamento das equipes e as promoções para técnico de enfermagem contribuíram significativamente neste momento. Outra intervenção para reconhecimento das equipes foi a liberação de um café reforçado.

\section{PRINCIPAIS RESULTADOS ALCANÇADOS}

Destacamos a tomada de decisão assertiva a respeito do Comitê de Crise, com o envolvimento de enfermeiros dedicados a realizar as modificações urgentes que a situação impõe.

\section{Limitações da experiência}

Embora tenham sido elencadas diversas ações para o atendimento do paciente, a limitação que se impõe é que, embora seja uma OSS, há dificuldades financeiras que precisam ser gerenciadas dentro deste contexto, pois não há conhecimento do tempo que a pandemia persistirá e da necessidade institucional para implementar novas mudanças.

\section{Contribuições para a prática}

Destaca-se a importância da equipe de enfermagem e seu preparo para gerenciar novos espaços, alocar recursos e treinar a equipe no atendimento direto ao paciente.

\section{CONCLUSÃO}

Apresentamos as principais mudanças na estrutura e nos processos de trabalho instituídos no hospital em questão para o atendimento do paciente suspeito ou confirmado com COVID-19. Embora muitas mudanças tenham sido feitas priorizando o paciente, poucas foram em relação à equipe de atendimento direto. Destacamos a importância de se criar espaços terapêuticos e apoio psicológico para a equipe; comunicação positiva evitando notícias falsas; momentos de pausa durante o plantão para o restabelecimento da condição emocional e alinhamento de informações para evitar que haja insegurança sobre os resultados institucionais.

A situação vivenciada leva a uma reflexão referente a importância da percepção da liderança de enfermagem não só nos aspectos técnicos para garantir um melhor atendimento aos pacientes com suspeita ou confirmados para COVID-19, mas também mostra a importância de se ter um olhar sobre como estão as condições físicas e emocionais dos profissionais que estão prestando a assistência direta.

CONTRIBUIÇÕES DOS AUTORES: Paula Maria Corrêa de Gouveia Araujo: concepção e/ou desenho do estudo ,redação e/ou revisão crítica do manuscrito e aprovação da versão final a ser publicada; Elena Bohomol: redação e/ou revisão crítica do manuscrito e aprovação da versão final a ser publicada; Tereza Aparecida Benjamim Teixeira: aprovação da versão final a ser publicada

AGRADECIMENTOS: $\grave{A}$ Diretoria Administrativa e todos os colaboradores do Hospital Santa Marcelina de Itaquaquecetuba.

\section{REFERÊNCIAS}

1. Wang C, Horby PW. Hayden FG, Gao GF. A novel coronavirus outbreak of global health concern. The lancet [Internet]. 2020 Feb 15 [cited 2020 Apr 29];395 Available from: https://www.ncbi.nlm.nih.gov/pubmed/31986257.

2. Cui J, Li F, Shi ZL. Origin and evolution of pathogenic coronaviruses. Nat Rev Microbiol [Internet]. 201903 [cited 2020 Apr 29]:17(3) Available from: https://www.ncbi.nlm.nih.gov/pubmed/30531947.

3. Folha informativa ? COVID-19 (doença causada pelo novo coronavirus) [Internet]. [place unknown]; 2020 Apr 29 [cited 2020 Apr 29]. Available from: https://www.paho.org/bra/index.php?option=com_content\&view=articlefid=6101: COVID19\&Itemid=875

4. IHR Procedures concerning public health emergencies of international concern (PHEIC) [Internet]. [place unknown]; 2020 Apr 30 [cited 2020 Apr 29]. Available from: https://www.who.int/ihr/procedures/pheic/en/.

5. Brasil. Portaria n.356, de 11 de março de 2020. Dispõe sobre a regulamentação e operacionalização do disposto na Lei no 13.979, de 6 de fevereiro de 2020, que estabelece as medidas para enfrentamento da emergência de saúde pública de importância internacional decorrente do coronavirus (COVID-19). Disponivel em: http://www.in.gov.br/en/web/ dou/-/portaria-n-356-de-11-de-marco-de-2020-247538346.

6. Chen Q, Liang M, Li Y, Guo J, Fei D, Wang L, et al. Mental health care for medical staff in China during the COVID-19 outbreak. Lancet Psychiatry [Internet]. 2020 Feb 19 [cited 2020 Apr 6];7(4):el5-el6. Available from: https://www.ncbi.nlm.nih.gov/pubmed/32085839.

7. Zhenyu Li, Ge J, Yang M, Feng J, Qiao M, Jiang R, et al. Vicarious traumatization in the general public, members, and non-members of medical teams aiding in COVID-19 control. Brain, Behavior, and Immunity [Internet]. 2020 Mar 09 [cited 2020 Apr 6]; Available from: https://www.sciencedirect.com/science/article/pii/S0889159120303093.

8. Machado MH, Wermelinger M, Vieira M, de Oliveira E, Lemos W. Filho WA, et al. Aspectos Gerais da Formação da Enfermagem: o Perfil da Formação dos Enfermeiros,Técnicos e Auxiliares. Enferm.foco [Internet] 2016 [cited 2020 Apr 6];7(especial).v7.nESP.687. Available from: http://revista.cofen.gov.br/index.php/enfermagem/article/view/687/297.

9. Isoldi DMR, Candido MCFS, Simpson CA, Silva FS. Fatores Relacionados às Ocorrências Éticas na Enfermagem Frente a Erros de Medicação. Revista Brasileira de Ciências da Saúde [Internet]. 2017 [cited 2020 Apr 29];21(4):369-388. Available from: http://periodicos.ufpb.br/ojs2/index. php/rbcs. 